\title{
Nonlinear energy pooling in nanophotonic materials
}

\author{
David L. Andrews* and David S. Bradshaw \\ Nanostructures and Photomolecular Systems, School of Chemical Sciences, \\ University of East Anglia, Norwich, NR4 7TJ, U.K.
}

\begin{abstract}
Recently there has been considerable interest in the construction of photoactive organic materials designed to exhibit novel forms of optical nonlinearity. By exploiting the unique properties of these nanomaterials at high levels of photon flux, new possibilities emerge for applications in energy harvesting, low-threshold lasing, quantum logic devices, photodynamic therapy, etc. In particular, a detailed appraisal of the theory spotlights novel mechanisms for directed energy transfer and energy pooling in nanophotonic dendrimers. Characterized by a nonlinear dependence on the optical irradiance, these mechanisms fall into two classes: (a) those where two-photon absorption by individual donors is followed by transfer of the sum energy to the acceptor; (b) where the excitation of two electronically distinct but neighbouring donor groups is followed by a collective migration of their energy to a suitable acceptor. In each case these transfer processes are subject to minor dissipative losses, associated with intramolecular vibrational relaxation in the donor species. In this paper we describe in detail the balance of factors and the constraints that determines the favored mechanism, which include the excitation statistics, structure of the energy levels, selection rules, molecular architecture, the distribution of donors and acceptors, spectral overlap and coherence factors. Knowledge of these factors and the means for their optimization offers fresh insights into nanophotonic characteristics, and informs strategies for the design of new photoactive materials.
\end{abstract}

Keywords: Organic optoelectronics, dendrimers, nanophotonics, photophysics, light harvesting, energy transfer, nonlinear optics, multiphoton absorption

\section{INTRODUCTION}

The elucidation of detailed principles for electronic energy flow in multichromophore polymers has led to a proliferation of new energy-harvesting materials tailored for a host of nanophotonic applications. ${ }^{1-3}$ Principal amongst these new materials are dendrimeric polymers - multiply-branched structures of essentially fractal geometry - alongside many other schematically related multichromophore assemblies. ${ }^{4-6}$ Such materials are highly effective in the capture of optical radiation, as a result of their multiplicity of antenna chromophores and the efficiency of resonance energy transfer (RET) mechanisms for the intramolecular channeling of energy to an acceptor core. Applications already range from photodynamic cancer therapy to organic light-emitting diodes.

It has now emerged that, in the relevant high intensity regime, suitably designed dendrimer materials (see for example figure 1) can exhibit two quite different types of mechanism for channeling excitation energy to an acceptor which is optically transparent at the input frequency. ${ }^{7-10}$ Both mechanisms are associated with two-photon absorption within the peripheral antenna region of the dendrimer. This may result in the optical excitation of either a single donor, or a pair of donor chromophores. In the former case, initiated by two-photon absorption at a donor, the subsequent mechanism ${ }^{11}$ entails two-photon resonance energy transfer (TPRET) directly to a neighboring acceptor;

$$
\mathrm{A}^{* *}+\mathrm{B} \stackrel{\text { TPRET }}{\longrightarrow} \mathrm{A}+\mathrm{B}^{*}
$$

where the two-star superscript denotes a two-photon excited state of the donor. The probability for fulfilling the initial conditions for this mechanism (i.e. for the donors to exhibit two-photon absorption) is enhanced at high levels of optical input. In the second case the mechanism is a twin-donor process ${ }^{12}$ which, following initial one-photon excitations of two electronically distinct donors ( $\mathrm{A}$ and $\mathrm{A}^{\prime}$ ), results in energy pooling i.e. a collective migration of donor energies to an acceptor chromophore, B. This mechanism also becomes effective under high intensity laser light due to the 


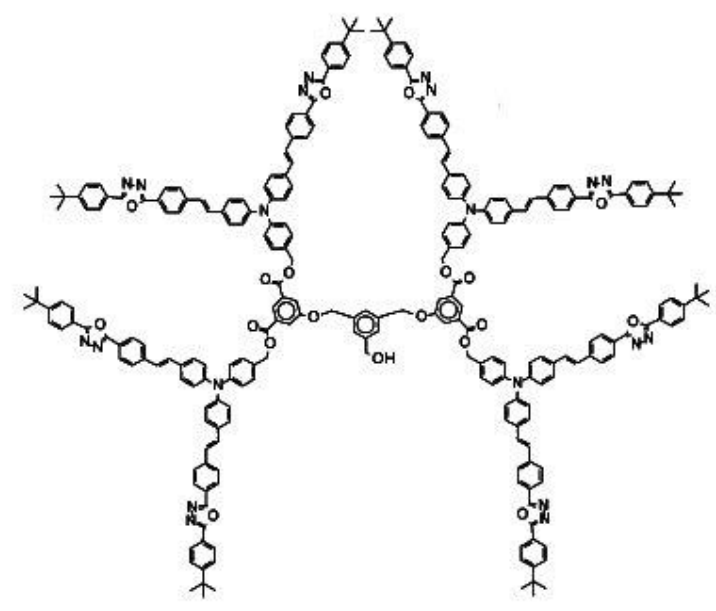

Fig. 1. Second generation two-photon absorbing dendrimer, adapted from Ref. 7.

enhanced probability of simultaneously exciting two donor chromophores within close proximity of each other and the acceptor. The overall process of energy pooling can be represented as;

$$
\mathrm{A}^{*}+\mathrm{B}+\mathrm{A}^{*^{*}} \stackrel{\text { Pooling }}{\longrightarrow} \mathrm{A}+\mathrm{B}^{*}+\mathrm{A}^{\prime}
$$

To expedite future progress in the development of optically nonlinear light-harvesting systems, it is clearly necessary to ascertain the means of differentiating, optimizing and exploiting the mechanisms available for energy capture. ${ }^{13-17}$ Since the mechanisms that are available to mediate energy harvesting under conditions of high photon flux differ markedly from those available at lower intensities, it is our aim to secure a through understanding of the principles that apply to systems specifically designed for operation at high levels of laser intensity. In this paper we describe in detail the balance of factors and the constraints that determines the favored mechanism for these forms of optical nonlinearity, which include: the excitation statistics, structure of the energy levels; laser coherence factors; chromophore selection rules and architecture; possibilities for the formation of delocalized excitons; spectral overlap; and the overall distribution of donors and acceptors. We begin by eliciting key components of the energy kinetics involved in each mechanism for nonlinear light-harvesting.

\section{TWO-PHOTON RESONANCE ENERGY TRANSFER MECHANISMS}

A theoretical representation based on molecular quantum electrodynamics (QED) can be used to derive an expression for the rate of a complete TPRET process, initiated by two-photon excitation of a single donor, as schematically illustrated by the energy scheme of figure 2. To determine the detailed kinetics we need to consider the mechanism of the RET step and accommodate statistical features associated with the initial excitation and donor conditions. Determining factors are the number of donors contained within the laser focal volume, the probability of a donor being two-photon excited, and the rate of energy transfer to the acceptor i.e. the RET step that completes the mechanism. For the ensuing analysis the relevant population factor is simply the number of donors within the focal volume, $N_{A}$ (assuming normal conditions, i.e. below the saturation limit, where the ground-state population is very similar to $N_{A}$ ) and, defining the number of suitably excited donors within the focal volume as $N_{2 A}$, the probability of two-photon excitation of a donor species under steady-state conditions is;

$$
\frac{N_{2 A}}{N_{A}} \simeq \frac{k_{A}^{2 \leftarrow 0}}{k_{A}^{0 \leftarrow 2}}
$$


where $k_{A}^{2 \leftarrow 0}$ is the two-photon absorption rate constant of the donor chromophore $A$ (dependent on the input intensity at the appropriate frequency) and $k_{A}^{0 \leftarrow 2}$ is the two-photon de-excitation rate constant. The former is derived by the

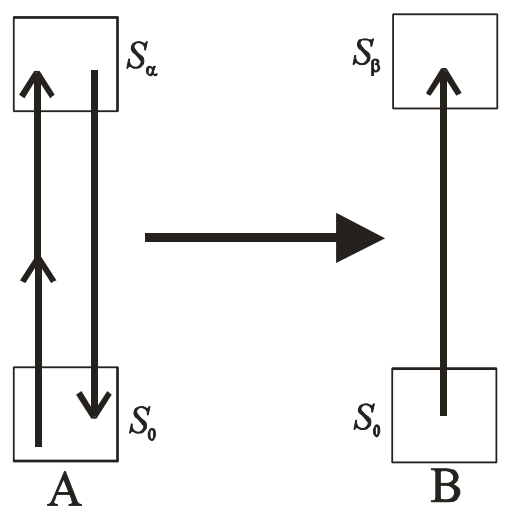

Fig. 2. Energy scheme for TPRET. $S_{0}$ represents the donor ground electronic state and its vibrational manifold; $S_{\alpha}$ denotes a higher electronic state. Vertical arrows represent transitions; the horizontal arrow denotes excitation transfer.

application of molecular QED leading to the following rotationally-averaged result, cast in terms of chromophore properties;

$$
\begin{aligned}
\left\langle k_{A}^{2 \leftarrow 0}\right\rangle= & \frac{\pi\left\langle I^{2}\right\rangle}{120 \hbar}\left(\frac{1}{\varepsilon_{0} c}\right)^{2}\left\{\left(2|\mathbf{e} \cdot \mathbf{e}|^{2}-1\right) \alpha_{i i}^{\alpha 0(A)}\left(\omega_{0}, \omega_{0}\right) \bar{\alpha}_{j j}^{\alpha 0(A)}\left(\omega_{0}, \omega_{0}\right)\right. \\
& \left.-\left(|\mathbf{e} \cdot \mathbf{e}|^{2}-3\right) \alpha_{i j}^{\alpha 0(A)}\left(\omega_{0}, \omega_{0}\right) \bar{\alpha}_{i j}^{\alpha 0(A)}\left(\omega_{0}, \omega_{0}\right)\right\} \rho_{f}^{A^{* *}} .
\end{aligned}
$$

Here $I$ is the irradiance, $\omega_{0}$ is the optical input circular frequency, $\rho_{f}^{A^{* *}}$ is the density of states for the excited state, reflecting vibrational broadening, and $\mathbf{e} \cdot \mathbf{e}$ is the self-product of the laser polarisation unit vector, which equates to 0 and 1 for circular and plane polarizations, respectively. Also $\alpha_{i j}$ is the generalized two-photon response tensor, of the general form;

$$
\alpha_{j k}^{f i(\xi)}\left(\mp \omega_{1}, \mp \omega_{2}\right)=\sum_{\zeta}\left\{\frac{\mu_{j}^{f \zeta(\xi)} \mu_{k}^{\zeta i(\xi)}}{E_{i \zeta} \pm \hbar \omega_{1}}+\frac{\mu_{k}^{f \zeta(\xi)} \mu_{j}^{\zeta i(\xi)}}{E_{i \zeta} \pm \hbar \omega_{2}}\right\}
$$

In equation (3), $i, \zeta$ and $f$ are the initial, virtual and final states respectively through which chromophore $\xi$ progresses. Note for conciseness the frequency dependence of the $\alpha_{i j}^{a b}$ factors is now implicit and follows from the superscripts. The dissipative effect of internal vibrational redistribution (IVR) does not itself feature in the electronic inter-state kinetics, though the associated red-shift in the emitted radiation is necessarily apparent and emerges in the following. For the single-step RET which delivers energy to the acceptor we have the familiar result from second-order perturbation theory; ${ }^{18}$

$$
\Gamma_{R E T}^{B \leftarrow A}=\frac{2 \pi}{\hbar}\left|\mu_{i}^{0 \alpha(A)} V_{i j}\left(\omega^{\prime}, \mathbf{R}\right) \mu_{j}^{\beta 0(B)}\right|^{2} \rho_{f}^{B}
$$

Here $\omega^{\prime}$ corresponds to the donor emission frequency, where $\omega^{\prime} \leq 2 \omega_{0} ; V_{i j}$ defines the electric dipole-electric dipole coupling tensor, given generally by $;^{11}$ 


$$
V_{j k}\left(\omega_{1}, \mathbf{R}\right)=\frac{\exp \left(\frac{\mathrm{i} \omega_{1} R}{c}\right)}{4 \pi \varepsilon_{0} R^{3}}\left\{\left(\delta_{j k}-3 \hat{R}_{j} \hat{R}_{k}\right)-\left(\frac{\mathrm{i} \omega_{1} R}{c}\right)\left(\delta_{j k}-3 \hat{R}_{j} \hat{R}_{k}\right)-\left(\frac{\omega_{1} R}{c}\right)^{2}\left(\delta_{j k}-\hat{R}_{j} \hat{R}_{k}\right)\right\},
$$

and the donor-acceptor displacement vector is defined as $\mathbf{R}=\mathbf{R}_{B}-\mathbf{R}_{A}$. Thus the total rate equation for a two-photon resonance energy transfer process emerges as;

$$
\begin{aligned}
\left\langle\Gamma^{\text {TPRET }}\right\rangle= & \frac{N_{A} g_{11}^{(2)}}{60}\left(\frac{\langle I\rangle \pi}{\varepsilon_{0} c h}\right)^{2}\left\{\left(2|\mathbf{e} \cdot \mathbf{e}|^{2}-1\right) \alpha_{i i}^{\alpha 0(A)} \bar{\alpha}_{i j}^{\alpha 0(A)}-\left(|\mathbf{e} \cdot \mathbf{e}|^{2}-3\right) \alpha_{i j}^{\alpha 0(A)} \bar{\alpha}_{i j}^{\alpha 0(A)}\right\} \\
& \times\left|\mu_{i}^{0 \alpha(A)} V_{i j}\left(\omega^{\prime}, \mathbf{R}\right) \mu_{j}^{\beta 0(B)}\right|^{2} \rho_{f}^{A^{* * *}} \rho_{f}^{B},
\end{aligned}
$$

where $g_{11}^{(2)}$ is the single-site degree of second-order optical coherence and the frequency dependence of the molecular tensors is implicit. Equation (6) will serve as a basis for judging the relative efficiency of two-photon and energy pooling energy transfer mechanisms; the latter is the subject of the next section.

\section{TWIN-DONOR ENERGY POOLING MECHANISMS}

In contrast to optically linear light harvesting, ${ }^{15}$ twin-donor energy pooling comprises two sub-mechanisms. ${ }^{19}$ These are defined as; (a) the cooperative mechanism, where the initial one-photon excitations are followed by RET from both donors directly to the acceptor, and (b) the accretive mechanism, where the initial excitation energy of one donor is passed to its partner and the sum of the two excitations is transferred to the acceptor. These two sub-mechanisms are illustrated by the energy schemes of figures 3 and 4. To construct a rate equation for the overall pooling process, accommodating both sub-mechanisms, a similar development to that used in the last section is employed, i.e. determining factors include; the number of donors contained within the laser focal volume, the probability of two such donors being simultaneously excited and the rate of twin-donor energy transfer to the acceptor.

First consider the probability of satisfying the initial conditions for excitation of a donor pair. The number of pairs at the laser focus, under standard conditions, is taken to be $\frac{1}{2} N_{A}\left(N_{A}-1\right)$, and if $N_{1 A}$ signifies the number of donors in the relevant electronic excited state, the probability of both partners in any one pair being excited is the square of $N_{1 A} / N_{A}$,

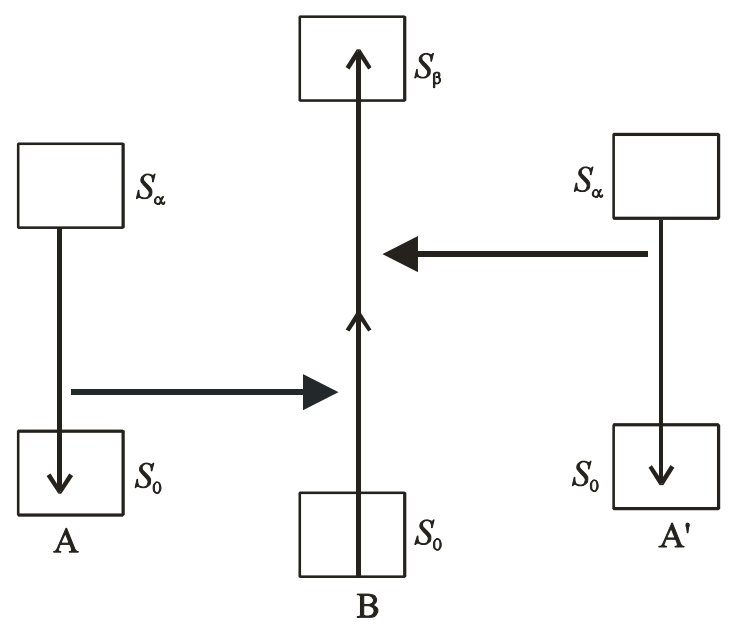

Fig. 3. Energy scheme for cooperative energy pooling: as in figure 2, with $S_{\beta}$ denoting a higher electronic state of the acceptor, B, and its associated manifold; $S_{a}$ represents a virtual state. 


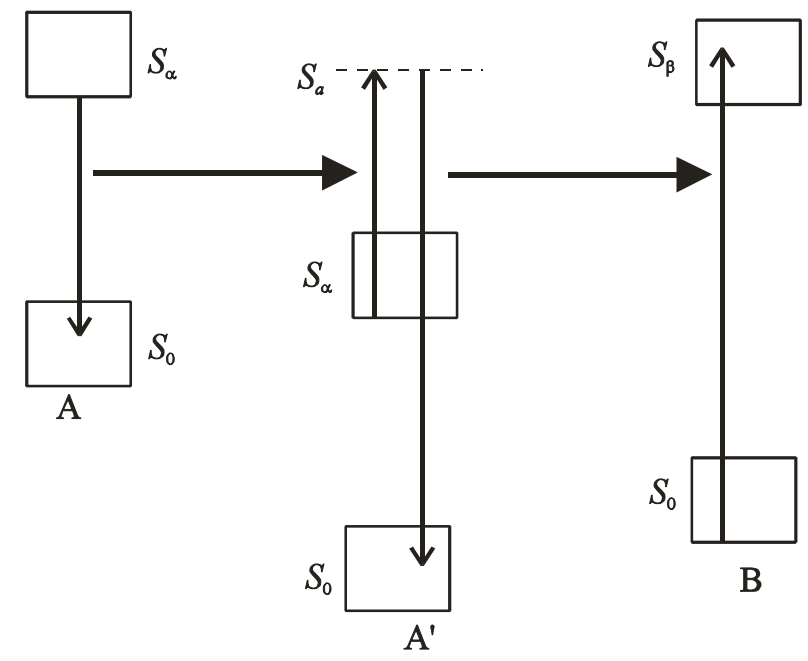

Fig. 4. Energy scheme for accretive energy pooling, where $A$ and $A^{\prime}$ are interchangeable.

assuming the decay lifetime is short compared to the laser pulse duration. Under such steady-state conditions the latter factor is given by;

$$
\frac{N_{1 A}}{N_{A}} \simeq \frac{k_{A}^{1 \leftarrow 0}}{k_{A}^{0 \leftarrow 1}},
$$

with $k_{A}^{1 \leftarrow 0}$ and $k_{A}^{0 \leftarrow 1}$ representing the one-photon absorption and de-excitation rate constants respectively. The former are again derived from molecular QED as follows;

$$
k_{A}^{1 \leftarrow 0}=\frac{\pi I}{3 \hbar c \varepsilon_{0}}\left|\boldsymbol{\mu}^{\alpha 0(A)}\right|^{2} \rho_{f}^{A^{*}},
$$

where $\mu^{\alpha 0}$ is the transition electric dipole moment connecting the donor ground state and excited state. Inserting equation (8) into (7);

$$
\frac{N_{1 A}}{N_{A}}=\frac{\pi I}{3 \hbar c \varepsilon_{0} k_{A}^{0 \leftarrow 1}}\left|\boldsymbol{\mu}^{\alpha 0(A)}\right|^{2} \rho_{f}^{A^{*}}
$$

Turning to the RET step, we have to consider both energy pooling sub-mechanisms. As shown in earlier work ${ }^{12,19}$ the results emerge from fourth-order perturbation theory in the form of the following rate equation;

$$
\begin{aligned}
\Gamma_{R E T}^{B \leftarrow A, A^{\prime}} & =\frac{2 \pi}{\hbar} \mid \mu_{i}^{0 \alpha(A)} V_{i j}(\omega, \mathbf{R}) \alpha_{j k}^{\beta 0(B)}(-\omega,-\omega) V_{k l}\left(\omega, \mathbf{R}^{\prime}\right) \mu_{l}^{0 \alpha\left(A^{\prime}\right)} \\
& +\left.2 \mu_{i}^{0 \alpha(A)} V_{i j}\left(\omega, \mathbf{R}^{\prime \prime}\right) \alpha_{j k}^{0 \alpha\left(A^{\prime}\right)}\left(\omega^{\prime},-\omega\right) V_{k l}\left(\omega^{\prime}, \mathbf{R}^{\prime}\right) \mu_{l}^{\beta 0(B)}\right|^{2} \rho_{f}^{B} .
\end{aligned}
$$

Here the first and second terms in the modulus relate to the cooperative and accretive sub-mechanisms, respectively, and each is cast in Cartesian components using the implied summation convention for repeated indices. Also $\omega$ corresponds to the donor emission frequency, where $\omega \leq \omega_{0}$ due to excited state IVR, the displacement vectors are defined as $\mathbf{R}^{\prime}=\mathbf{R}_{B}-\mathbf{R}_{A^{\prime}}, \mathbf{R}^{\prime \prime}=\mathbf{R}_{A^{\prime}}-\mathbf{R}_{A}$ and the factor of 2 at the front of the second term of equation (10) reflects the 
interchangeability of $A$ and $A^{\prime}$. Putting together the various factors delineated above, the ensemble averaged rate equation for the energy pooling process is as follows;

$$
\begin{aligned}
\left\langle\Gamma^{\text {Pooling }}\right\rangle & =N_{A}\left(N_{A}-1\right)\left(\frac{\pi^{\frac{3}{2}}}{3 \hbar^{\frac{3}{2}} c \varepsilon_{0} k_{A}^{0 \leftarrow 1}}\right)^{2}\langle I\rangle^{2}\left|\mu^{\alpha 0(A)}\right|^{4}\left(\rho_{f}^{A^{*}}\right)^{2} \rho_{f}^{B} \\
& \times\left|\mu_{i}^{0 \alpha(A)} V_{i j}(\omega, \mathbf{R}) \alpha_{j k}^{\beta 0(B)} V_{k l}\left(\omega, \mathbf{R}^{\prime}\right) \mu_{l}^{0 \alpha\left(A^{\prime}\right)}+2 \mu_{i}^{0 \alpha(A)} V_{i j}\left(\omega, \mathbf{R}^{\prime \prime}\right) \alpha_{j k}^{0 \alpha\left(A^{\prime}\right)} V_{k l}\left(\omega^{\prime}, \mathbf{R}^{\prime}\right) \mu_{l}^{\beta 0(B)}\right|^{2} .
\end{aligned}
$$

The excitation dynamics of the chemically equivalent $A$ and $A^{\prime}$ are for simplicity taken to be identical in the initial conditions, but the decay processes are necessarily differentiated in the RET step to accommodate potentially differing orientations in space.

\section{KEY CRITERIA}

The starting point for an exploration of the structural and electronic design factors, and their bearing on the relative efficiencies of each mechanism, we take the ratio of equations (11) and (6), to produce the result;

$$
\begin{aligned}
\frac{\left\langle\Gamma^{\text {Pooling }}\right\rangle}{\left\langle\Gamma^{\text {TPRET }}\right\rangle}= & \frac{20 \pi\left(N_{1 A}-1\right)\left(\rho_{f}^{A^{*}}\right)^{2}}{3 \hbar g_{11}^{(2)} \rho_{f}^{A^{* *}}}\left(\frac{k_{A}^{0 \leftarrow 2}}{\left(k_{A}^{0 \leftarrow-1}\right)^{2}}\right)\left(\frac{\left|\boldsymbol{\mu}^{\alpha 0(A)}\right|^{4}}{\left(2|\mathbf{e} \cdot \mathbf{e}|^{2}-1\right) \alpha_{i i}^{\alpha 0(A)} \bar{\alpha}_{j j}^{\alpha 0(A)}-\left(|\mathbf{e} \cdot \mathbf{e}|^{2}-3\right) \alpha_{i j}^{\alpha 0(A)} \bar{\alpha}_{i j}^{\alpha 0(A)}}\right) \\
& \times\left(\frac{\left|\mu_{i}^{0 \alpha(A)} V_{i j}(\omega, \mathbf{R}) \alpha_{j k}^{\beta 0(B)} V_{k l}\left(\omega, \mathbf{R}^{\prime}\right) \mu_{l}^{0 \alpha(A)}+2 \mu_{i}^{0 \alpha(A)} V_{i j}\left(\omega, \mathbf{R}^{\prime \prime}\right) \alpha_{j k}^{0 \alpha\left(A^{\prime}\right)} V_{k l}\left(\omega^{\prime}, \mathbf{R}^{\prime}\right) \mu_{l}^{\beta 0(B)}\right|^{2}}{\left|\mu_{i}^{0 \alpha(A)} V_{i j}\left(\omega^{\prime}, \mathbf{R}\right) \mu_{j}^{\beta 0(B)}\right|^{2}}\right) .
\end{aligned}
$$

In the following, attention focuses on a number of key facets of the above result. These are; spectroscopic factors, structural factors and quantum effects.

\section{A. Spectroscopic factors}

The detailed form of spectral overlap associated with each mechanism is a matter of considerable interest. As in conventional single-donor energy transfer, to determine a rate equation connected to Förster theory for TPRET mechanisms requires the consideration of spectral overlap between the donor fluorescence spectrum and the dispersive absorption cross-section of the acceptor - the detailed form depending on distance, and in the short range leading to the familiar Förster result. The QED formulation of this rate equation is given by; ${ }^{18}$

$$
\Gamma_{R E T}^{B \leftarrow A}=\frac{9}{8 \pi c^{2} \tau_{A}} \int_{0}^{\infty} F_{A}\left(\omega^{\prime}\right) \sigma_{B}\left(\omega^{\prime}\right) \omega^{\prime 2} g^{v a c}\left(\omega^{\prime}, \mathbf{R}\right) d \omega^{\prime} .
$$

Equation (13) includes the radiative lifetime of the donor, $\tau_{A}$, the cross-section of the acceptor absorption and donor emission spectra, these are defined generally as;

$$
\sigma_{B}(\omega)=\frac{\pi \omega}{3 \varepsilon_{0} c}\left|\mu^{0 \beta(B)}\right|^{2} \sum_{m, p} \rho_{B}^{(m)}\left|\left\langle\varphi_{B^{*}}^{(p)} \mid \varphi_{B}^{(m)}\right\rangle\right|^{2} \delta\left(e_{B_{p}^{*}}-e_{B_{m}}-\hbar \omega\right),
$$

and

$$
F_{A}(\omega)=\frac{\omega^{3} \tau_{A}}{3 \varepsilon_{0} \pi \hbar c^{3}}\left|\boldsymbol{\mu}^{\alpha 0(A)}\right|^{2} \sum_{n, r} \rho_{A^{*}}^{(n)}\left|\left\langle\varphi_{A}^{(r)} \mid \varphi_{A^{*}}^{(n)}\right\rangle\right|^{2} \delta\left(e_{A_{n}^{*}}-e_{A_{r}}-\hbar \omega\right),
$$


respectively. Here, $\rho$ is the population distribution function of the initial vibrational states of the specified species, each of the indices $n, r, m, p$ specifies the set of vibrational etc. sub-levels of the transfer species, the energies of the initial and final state of each species are included in the energy-conserving delta function. In detail $F_{A}(\omega)$ is determined by the exit state of $A$, which is a consequence of initial excitation and subsequent IVR. Also included in equation (13) is $g^{v a c}$ which emerges from equation (5) and is generally given by;

$$
g^{v a c}(\omega, \mathbf{R})=\eta_{3}^{2} \frac{c^{6}}{\omega^{6} R^{6}}+\left(\eta_{3}^{2}-2 \eta_{1} \eta_{3}\right) \frac{c^{4}}{\omega^{4} R^{4}}+\eta_{1}^{2} \frac{c^{2}}{\omega^{2} R^{2}}
$$

where $\eta_{q}$ are the orientational factors and expressible as;

$$
\eta_{q}=\left(\hat{\boldsymbol{\mu}}_{A} \cdot \hat{\boldsymbol{\mu}}_{B}\right)-q\left(\hat{\mathbf{R}} \cdot \hat{\boldsymbol{\mu}}_{A}\right)\left(\hat{\mathbf{R}} \cdot \hat{\boldsymbol{\mu}}_{B}\right) \quad(q=1,3)
$$

In the short range equation (13) takes the form of the Förster rate, which is given as follows;

$$
\Gamma_{R E T}^{B \leftarrow A}=\frac{9 c^{4} \eta_{3}^{2}}{8 \pi \tau_{A} R^{6}} \int_{0}^{\infty} F_{A}\left(\omega^{\prime}\right) \sigma_{B}\left(\omega^{\prime}\right) \omega^{\prime-4} d \omega^{\prime}
$$

The form of spectral overlap associated with the energy pooling processes, i.e. twin-donor transfer, is more complex due to the energy transferal of $\hbar \omega$ from two donor species to an acceptor and differs for each of the sub-mechanisms. In the case of cooperative transfer the following rate is found;

$$
\begin{aligned}
\Gamma_{R E T, \text { Coop }}^{B \leftarrow A, A^{\prime}} & =\frac{9}{64 \pi^{2} c^{4} \tau_{A} \tau_{A^{\prime}}} \iint \omega^{2}\left(\omega^{\prime}-\omega\right)^{2} F_{A}(\omega) F_{A^{\prime}}\left(\omega^{\prime}-\omega\right) \tilde{\sigma}_{B}\left(\omega, \omega^{\prime}-\omega\right) \\
& \times g^{v a c}(\omega, \mathbf{R}) g^{v a c}\left(\omega^{\prime}-\omega, \mathbf{R}^{\prime}\right) d \omega d \omega^{\prime}
\end{aligned}
$$

where $F_{A}$ and $F_{A^{\prime}}$ are both given by equation (15), $g^{v a c}$ by equation (16) and the cross-section of the acceptor two-photon absorption, $\tilde{\sigma}_{B}\left(\omega, \omega^{\prime}-\omega\right)$, is defined as follows;

$$
\tilde{\sigma}_{B}\left(\omega, \omega^{\prime}-\omega\right)=\frac{\pi \hbar \omega\left(\omega^{\prime}-\omega\right)}{2 \varepsilon_{0}^{2} c^{2}}\left|\boldsymbol{\alpha}^{B}\left(\omega, \omega^{\prime}-\omega\right)\right|^{2} \sum_{m, p} \rho_{B}^{(m)}\left|\left\langle\varphi_{B^{*}}^{(p)} \mid \varphi_{B}^{(m)}\right\rangle\right|^{2} \delta\left(e_{B_{p}^{*}}-e_{B_{m}}-\hbar \omega^{\prime}\right) .
$$

The rate equation for the accretive sub-mechanism is slightly different to the form of the cooperative result of equation (19) and is found to be;

$$
\begin{aligned}
\Gamma_{R E T, A c c}^{B \leftarrow A, A^{\prime}} & =\frac{9}{64 \pi^{2} c^{4} \tau_{A} \tau_{A^{\prime}}} \iint \omega^{2} \omega^{\prime 2} F_{A}(\omega) S_{A^{\prime}}\left(\omega,-\omega^{\prime}\right) \sigma_{B}\left(\omega^{\prime}\right) \\
& \times g^{v a c}\left(\omega, \mathbf{R}^{\prime \prime}\right) g^{v a c}\left(\omega^{\prime}, \mathbf{R}^{\prime}\right) d \omega d \omega^{\prime}
\end{aligned}
$$

Here $\sigma_{B}\left(\omega^{\prime}\right)$ and $F_{A}(\omega)$ are given by equation (14) and (15) respectively. Equation (21) also includes $S_{A^{\prime}}\left(\omega,-\omega^{\prime}\right)$, the frequency-dependent anti-Stokes electronic Raman scattering (ASRS) cross-section of the $A^{\prime}$ chromophore, given by; 


$$
S_{A^{\prime}}\left(\omega,-\omega^{\prime}\right)=\frac{\omega \omega^{\prime 3} \tau_{A^{\prime}}}{2 \pi \varepsilon_{0}^{2} c^{4}}\left|\boldsymbol{\alpha}^{A^{\prime}}\left(\omega,-\omega^{\prime}\right)\right|^{2} \sum_{q, t} \rho_{A^{\prime \prime}}^{(q)}\left|\left\langle\varphi_{A^{\prime}}^{(t)} \mid \varphi_{A^{\prime}}^{(q)}\right\rangle\right|^{2} \delta\left(e_{A_{q}^{\prime \prime}}-e_{A_{t}^{\prime}}-\hbar\left(\omega^{\prime}-\omega\right)\right) .
$$

In the short range, considering equations (19) and (21), the total rate for the energy pooling process emerges as;

$$
\begin{aligned}
\Gamma_{R E T}^{B \leftarrow A, A^{\prime}}= & \frac{9 c^{8} \eta_{3}^{4}}{64 \pi^{2} \tau_{A} \tau_{A^{\prime}}}\left[\iint R^{-6} R^{\prime-6} \omega^{-4}\left(\omega^{\prime}-\omega\right)^{-4} F_{A}(\omega) F_{A^{\prime}}\left(\omega^{\prime}-\omega\right) \tilde{\sigma}_{B}\left(\omega^{\prime}\right)\right. \\
& \left.+2 R^{\prime-6} R^{\prime \prime-6} \omega^{-4} \omega^{\prime-4} F_{A}(\omega) S_{A^{\prime}}\left(\omega,-\omega^{\prime}\right) \sigma_{B}\left(\omega^{\prime}\right) d \omega d \omega^{\prime}\right],
\end{aligned}
$$

where the first and second term of the integrand correspond to the cooperative and accretive sub-mechanism respectively. Note again the double counting of the accretive term due to the interchangeability of $A$ and $A^{\prime}$.

\section{B. Structural factors}

Each of the mechanisms for optically nonlinear photoactivity entails a different form of interaction for the donor and acceptor units. The selection rules for one-photon and two-photon processes differ and, for a given system, one or more of the mechanisms or sub-mechanisms may be forbidden by local symmetry. The transition dipole moments, $\boldsymbol{\mu}^{a b}$, and transition tensors, $\boldsymbol{\alpha}^{a b}$, within each of the three mechanisms are associated with irreducible representations (irreps) of the site point group (as detailed in ref. 20). The nature of the associated donor and acceptor transitions imposes conditions on the validity of each mechanism. Generally, the direct product of the initial and final state representations must span at least one of the irrep components of the relevant transition tensors. An example is the accretive sub-mechanism, which is only symmetry-allowed if the donor decay transition has transformation properties replicated by a component of $D^{(1-)}$, i.e. the irrep for the initial photoabsorption. Furthermore, the same transition must have the transformation properties associated with a component of $D^{(0+)} \oplus D^{(1+)} \oplus D^{(2+)}$. The excitation transition dipole moment of the acceptor will also need to transform as one or more components of $D^{(1-)}$. The detailed form of the irrep components is governed by the local point group symmetry $D_{3 h}, D_{4 h}$ and $C_{2 v}$ in many dendrimeric materials. According to the chromophore architecture, specific conclusions can be drawn for each of two main classes of dendrimeric light-harvesting materials: (i) For systems of $D_{3 h}$ threefold symmetry for both donor and acceptor sites; when the direct product of the donor ground and excited state representations includes the irrep $E^{\prime}$, all of the discussed mechanisms are permitted - whereas only cooperative pooling is allowed when the same product spans $A_{2}^{\prime \prime}$. (Note the assumption of equivalence between the absorption and emission transition symmetries of the donor species). Many examples of this type of material are based on polyphenylethynl dendrimers. ${ }^{21,22}$ (ii) For systems based on $C_{2 v}$ and $D_{4 h}$ symmetry for the donor and acceptor species, respectively, all three mechanisms are symmetry allowed through transitions of $A_{1}, B_{1}$ and $B_{2}$ symmetry. In addition, both TPRET and accretive processes are allowed through $A_{2}$ transitions. For the acceptor, transitions with $A_{2 u}$ and $E_{u}$ symmetry permit the TPRET and accretive mechanisms, while $A_{1 g}, B_{1 g}, B_{2 g}$ and $E_{g}$ allow cooperative transfer. Examples of these systems are light-harvesting assemblies of porphyrin ${ }^{23,24}$ (one instance illustrated in figure 5) and multiporphyrin arrays. ${ }^{25}$

The overall chromophore layout also plays a role in determining the preferred energy transfer mechanism. Thus, for energy pooling processes in dendrimeric systems with a $D_{3 h}$ symmetric nodal motif, the cooperative sub-mechanism is promoted by the closer proximity of the donor and acceptor species in comparison to the donor-donor separation. This follows from the form of the coupling tensors in the two cases, $V_{i j}(\omega, \mathbf{R}) V_{k l}\left(\omega, \mathbf{R}^{\prime}\right)$ for the cooperative mechanism and $V_{i j}\left(\omega, \mathbf{R}^{\prime \prime}\right) V_{k l}\left(\omega, \mathbf{R}^{\prime}\right)$ for the accretive, bearing in mind that in the short-range, each $\mathbf{V}(\omega, \mathbf{R})$ has an overall dependence on $R^{-3}$, where $R$ is the magnitude of the displacement vector in the argument. As the number of donor chromophores around the acceptor increases the accretive sub-mechanism becomes of increasing importance as shown by the ratios of table 1 . 


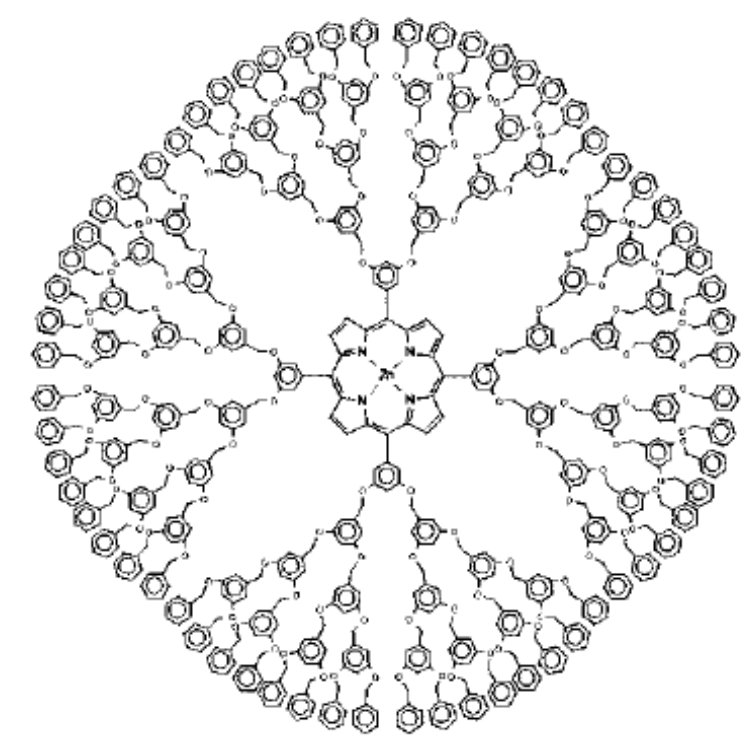

Fig. 5. Fourth generation dendrimer based on a zinc porphyrin core, adapted from ref. 23.

\begin{tabular}{|l|l|l|l|l|l|l|l|}
\hline$n$ & 3 & 4 & 5 & 6 & 7 & 8 & 9 \\
\hline acc:coop & 0.037 & 0.125 & 0.379 & 1.000 & 2.34 & 4.97 & 9.76 \\
\hline
\end{tabular}

Table 1. Relative importance of the accretive sub-mechanism for energy pooling from a ring of $n$ donors.

Finally, the detailed nanoscale architecture also has a considerable effect on the dominance of one energy pooling sub-mechanism over the other. ${ }^{26}$ To estimate the associated effects we can consider that all transition dipole moments and separation vectors are equivalent, and that components of each of the $\alpha$ tensors have similar magnitude to the corresponding polarisability components. Then, it is possible to write the intimidating expression within the final bracket of equation (12) in the greatly simplified form that follows;

$$
\frac{\Gamma^{\text {Pooling }}}{\Gamma^{\text {TPRET }}} \sim \frac{54 \alpha^{\prime 2}}{R^{6}}
$$

where $\alpha^{\prime}$ is a volume polarisability. Equation (24) shows that light-harvesting systems based on small, essentially nonpolarisable chromophores with tightly bound electrons (small $\alpha^{\prime}$ ) are generally dominated by TPRET, while energy pooling is favored by systems with tightly packed donors and acceptors (small separations). In the latter case specifically, cooperative transfer is favored for systems with a highly polarisable acceptor, accretive transfer for those with highly polarisable donors.

\section{Quantum effects}

In general, the initiation of TPRET requires only one excited donor and the initial energy deposition is localized to that species. Often, dendrimeric materials are designed with spacer units separating the donor and acceptor chromophores, so as to retain their distinct electronic integrity and preclude charge transfer. However, if two or more identical donor species within the proximity of each other are electronically coupled to any significant extent, an exciton may form. Excitons in this context ${ }^{27}$ are associated with an uncertainty in the location of the photon energy deposition and are generated when the number of excitations within a chromophore array is less than the number of donors it comprises. Hence energy pooling processes, which require two excited donor species, may engage three or more donors in excitonic states. Consider, for example, a threefold symmetric, nodal component of a dendrimer comprising chemically 
identical donors $\mathrm{A}, \mathrm{B}$ and $\mathrm{C}$ each at one corner of an equilateral triangle, with an acceptor, $\mathrm{D}$, at the centre. Both in TPRET and energy pooling a donor exciton intermediate can form, as is illustrated by the equation;

$$
\mathrm{A}+\mathrm{B}+\mathrm{C}+\mathrm{D}+2 \hbar \omega \stackrel{\text { Exciton formation }}{\longrightarrow}(\mathrm{A}+\mathrm{B}+\mathrm{C})^{* *}+\mathrm{D} \stackrel{R E T}{\longrightarrow} \mathrm{A}+\mathrm{B}+\mathrm{C}+\mathrm{D}^{*}
$$

Although associated in each case with the energy of two input photons, the exciton is recognized to have a different structure for the two processes. Specifically, $(A+B+C)^{* *}$ is one of two forms - either $\left(A^{* *}+B+C\right)$ and its permutations for TPRET, or $\left(\mathrm{A}^{*}+\mathrm{B}^{*}+\mathrm{C}\right)$ and permutations for energy pooling. ${ }^{28,29}$

The processes of energy pooling and TPRET lead from the same initial state to a final state in which the acceptor is electronically excited. However the dissipation of energy through IVR in the donor ensemble is different in the two cases so that, whereas these mechanisms may compete if both are allowed, they cannot display quantum interference. That is not the case, however, when we consider the two sub-mechanisms for energy pooling, because the two electronic couplings that each of these involves are concerted and not step-wise processes, as the QED calculations show. In other words the quantum pathways from the initial to the final state traverse only virtual states, in which energy losses are not sustained into or beyond the femtosecond timescale. In the numerator of the last bracketed terms in equation (12) the cooperative and accretive sub-mechanisms are represented by the first and second terms, respectively, and the result is multiplied by its complex conjugate. Thus it emerges that there is a cross-term representing the quantum interference of the two sub-mechanisms, which is of clear physical significance and entails interdependent chromophore separation vectors. In the unfolding technology of nanophotonic materials, the significance of such quantum interferences should not be underestimated.

\section{DISCUSSION}

In this paper we have begun to address the principles associated with a multitude of factors whose interplay determines the favored mechanism for optically nonlinear photoactivity. In general, nanomaterials of this kind are designed to expedite one specific mechanism. Examples of TPRET dendrimers are given in refs 7-10; recent examples of energy pooling materials can be found in refs 4-6, 21-25. Previously, it has not been generally recognized that the two mechanisms can operate in parallel, given suitably placed chromophore energy levels, and subject to the geometric and symmetry-based criteria detailed above. There is considerable scope to exploit this diversity of mechanisms, and it is our hope that as increasingly detailed principles emerge, these will inform and steer future efforts in the creation of light-harvesting nanomaterials. ${ }^{30}$

In conclusion, it is interesting to note that TPRET also operates in other quite different areas of application. One example is where RET is involved in two-photon three-dimensional imaging ${ }^{31}$ - a technique which is especially advantageous for biological specimens due to the enhanced depth profiling and reduced photolytic damage. Also, energy harvesting dendrimers are increasingly being developed for use in organic light-emitting diode materials. ${ }^{32}$ Furthermore, energy pooling porphyrin dendrimers have begun to find an application in laser photodynamic therapy (PDT) as photosensitisers ${ }^{33}$ - energy harvesting here leading to the photochemical destruction of cancer cells via generation of singlet oxygen. As results emerge in each of these and other new areas, the relative importance of TPRET and energy pooling as competing processes can also now be examined in the appropriate detailed context, using the results we have reported.

\section{ACKNOWLEDGMENT}

DSB is funded by a studentship from the Engineering and Physical Sciences Research Council. We thank Dr Luciana Dávila Romero for helpful comments on this work.

\section{REFERENCES}

1. D. L. Andrews and A. A. Demidov, ed. Resonance Energy Transfer, Wiley, Chichester, 1999.

2. P. R. Selvin, "The renaissance of fluorescence resonance energy transfer”, Nature Struct. Bio. 7, pp. 730-734, 2000. 
3. D. L. Andrews, "Optical energy harvesting materials", Introduction to Complex Mediums for Optics and Electromagnetics, ed. W. S. Weiglhofer and A. Lakhtakia, SPIE, Bellingham, Washington, pp. 141-163, 2003.

4. A. Archut and F. Vögtle, "Functional cascade molecules", Chem. Soc. Rev. 27, pp. 233-240, 1998.

5. A. Adronov and J. M. J. Fréchet, "Light-harvesting dendrimers", Chem. Commun., pp. 1701-1710, 2000.

6. V. Balzani, P. Ceroni, M. Maestri and V. Vicinelli, "Light-harvesting dendrimers", Curr. Opin. Chem. Biol. 7, pp. 657-665, 2003.

7. A. Adronov, J. M. J. Fréchet, G. S. He, K. S. Kim, S. J. Chung, J. Swiatkiewicz and P. N. Prasad, "Novel two-photon absorbing dendritic structures", Chem. Mater. 12, pp. 2838-2841, 2000.

8. G. S. He, T. C. Lin, Y. Cui, P. N. Prasad, D. Brousmiche, J. M. Serin and J. M. J. Fréchet, "Two-photon excited intramolecular energy transfer and light-harvesting effect in novel dendritic systems", Opt. Lett. 28, pp. 768-770, 2003.

9. D. Brousmiche, J. M. Serin, J. M. J. Fréchet, G. S. He, T. C. Lin, S. J. Chung and P. N. Prasad, "Fluorescence energy transfer in a novel two-photon absorbing system", J. Am. Chem. Soc. 125, pp. 1448-1449, 2003.

10. O. Mongin, J. Brunel, L. Porrès and M. Blanchard-Desce, "Synthesis and two-photon absorption of triphenylbenzene-cored dendritic chromophores", Tetrahedron Lett. 44, pp. 2813-2816, 2003.

11. P. Allcock and D. L. Andrews, “Two-photon fluorescence: resonance energy transfer”, J. Chem. Phys. 108, pp. 3089-3095, 1998.

12. R. D. Jenkins and D. L. Andrews, "Three-center systems for energy pooling: quantum electrodynamic theory”, J. Phys. Chem. A 102, pp. 10834-10842, 1998.

13. A. Bar-Haim and J. Klafter, "Geometric versus energetic competition in light-harvesting by dendrimers", J. Phys. Chem. B 102, pp. 1662-1664, 1998.

14. S. Tretiak, V. Chernyak and S. Mukamel, "Localized electronic excitations in phenylacetylene dendrimers", J. Phys. Chem. B 102, pp. 3310-3315, 1998.

15. A. Bar-Haim and J. Klafter, "Dendrimers as light-harvesting antennae”, J. Lumin. 76\&77, pp. 197-200, 1998.

16. Y. Wakabayashi, M. Tokeshi, D.-L. Jiang, T. Aida, T. Kitamori, "Long-term energy storage of dendrimers", J. Lumin. 83\&84, pp. 313-315, 1999.

17. K. Kondo, S. Yasuda, T. Sakaguchi and M. Miya, "The third-order optical non-linearity of the phenylethynylsubstituted benzene system”, J. Chem. Soc., Chem. Commun., pp. 55-56, 1995.

18. G. Juzeliūnas and D. L. Andrews, "Unified theory of radiative and radiationless energy transfer", Resonance Energy Transfer, ed. D. L. Andrews and A. A. Demidov, Wiley, Chichester, pp. 65-107, 1999.

19. R. D. Jenkins and D. L. Andrews, “Twin-donor systems for resonance energy transfer”, Chem. Phys. Lett. 301, pp. 235-240, 1999.

20. D. L. Andrews, "Symmetry characterization in molecular multiphoton spectroscopy", Spectrochimica Acta 46A, pp. 871-885, 1990.

21. M. R. Shortreed, S. F. Swallen, Z.-Y. Shi, W. Tan, Z. Xu, C. Devadoss, J. S. Moore and R. Kopelman, "Directed energy transfer funnels in dendrimeric antenna supermolecules ”, J. Phys. Chem. B 101, pp. 6318-6322, 1997.

22. Z. Xu and J. S. Moore, "Design and synthesis of a convergent and directional molecular antenna", Acta Polym. 45 , pp. 83-87, 1994.

23. M. S. Matos, J. Hofkens, W. Verheijen, F. C. De Schryver, S. Hecht, K. W. Pollak, J. M. J. Fréchet, B. Forier and W. Dehaen, "Effect of core structure on photophysical and hydrodynamic properties of porphyrin dendrimers", Macromolecules 33, pp. 2967-2973, 2000.

24. D. K. P. Ng, C. R. Chimie 6, "Dendritic phthalocyanines: synthesis, photophysical properties and aggregation behavior", pp. 903-910, 2003.

25. M. del Rosario Benites, T. E. Johnson, S. Weghorn, L. Yu, P. D. Rao, J. R. Diers, S. I. Yang, C. Kirmaier, D. F. Bocian, D. Holten and J. S. Lindsey, "Synthesis and properties of weakly coupled dendrimeric multiporphyrin light-harvesting arrays and hole-storage reservoirs", J. Mater. Chem. 12, pp. 65-80, 2002.

26. R. D. Jenkins and D. L. Andrews, "Orientation factors in three-centre energy pooling”, Phys. Chem. Chem. Phys. 2, pp. 2837-2843, 2000.

27. R. D. Jenkins and D. L. Andrews, "Multichromophore excitons and resonance energy transfer: molecular quantum electrodynamics", J. Chem. Phys. 118, pp. 3470-3479, 2003.

28. R. D. Jenkins and D. L. Andrews, "Exciton resonance energy transfer: effects of geometry and transition moment orientation in model photosystems", Photochem. Photobiol. Sci. 2, pp. 130-135, 2003.

29. R. D. Jenkins and D. L. Andrews, "Biexciton resonance energy transfer in a model photosystem", Photochem. Photobiol. Sci. 3, pp. 39-46, 2004. 
30. P. Ball and D. L. Andrews, "Light harvesting", Chem. World 1(3), 34-39, 2004.

31. C. Xu, W. Zipfel, J. B. Shear, R. M. Williams and W. W. Webb, "Multiphoton fluorescence excitation: new spectral windows for biological nonlinear microscopy”, Proc. Natl. Acad. Sci. USA 93, pp. 10763-10768, 1996.

32. A. W. Freeman, S. C. Koene, P. R. L. Malenfant, M. E. Thompson and J. M. J. Fréchet, "Dendrimer-containing light-emitting diodes: toward site-isolation of chromophores", J. Am. Chem. Soc. 122, pp. 12385-12386, 2000.

33. G.-D. Zhang, A. Harada, N. Nishiyama, D.-L. Jiang, H. Koyama, T. Aida and K. Kataoka, "Polyion complex micelles entrapping cationic dendrimer porphyrin: effective photosensitizer for photodynamic therapy of cancer", J. Control. Release 93, pp. 141-150, 2003.

*david.andrews@physics.org 